\title{
Neurografia do plexo braquial em ressonância magnética ponderada em difusão: Uma revisão da
}

\section{literatura}

\author{
Diffusion-Weighted MR Neurography of the Brachial Plexus: A literature review \\ Imagen de resonancia magnética ponderada por difusión del plexo braquial: Revisión de la
}

literatura

Recebido: 17/04/2021 | Revisado: 23/04/2021 | Aceito: 25/04/2021 | Publicado: 09/05/2021

Antonione Santos Bezerra Pinto

ORCID: https://orcid.org/0000-0002-6577-2816

Faculdade de Ciências Humanas, Exatas e da Saúde do Piauí, Brasil Instituto de Educação Superior do Vale do Parnaíba, Brasil

E-mail: antonione.pinto@iesvap.edu.br

André Luca Araújo de Sousa

ORCID: https://orcid.org/0000-0003-4876-9188

Universidade Estadual do Piauí, Brasil

E-mail: a.lucaaraujo10@gmail.com

Álvaro Xavier Franco

ORCID: https://orcid.org/0000-0003-3600-7290 Universidade Federal do Ceará, Brasil E-mail: alv_17@yahoo.com.br

Francisco Rafael Oliveira da Silva

ORCID: https://orcid.org/0000-0002-9550-4898 Universidade Federal do Ceará, Brasil E-mail: rafael7personal82@gmail.com

Regina Paula Soares Diego

ORCID: https://orcid.org/0000-0002-9606-0598 Universidade Federal do Ceará, Brasil E-mail: reginadiego@hotmail.com

Uziel Nunes Silva

ORCID: https://orcid.org/0000-0002-0153-8596 Faculdade de Ciências Humanas, Exatas e da Saúde do Piauí, Brasil Instituto de Educação Superior do Vale do Parnaíba, Brasil E-mail: uziel.silva@iesvap.edu.br

\begin{abstract}
Resumo
O plexo braquial é formado pela união dos ramos ventrais das raízes C5-T1 e, ocasionalmente, das raízes C4 e T2, onde as causas de lesões traumáticas mais frequentes envolvem acidentes de trânsito, principalmente colisão por motocicletas. O diagnóstico preciso do nível da lesão e grau de comprometimento tem fundamental importância prognóstica e determina a conduta terapêutica para cada caso clínico. Objetivando avaliar as limitações e perspectivas da aplicação da neurografia por difusão em RM para geração de imagens do plexo braquial em diferentes situações patológicas, realizou-se o levantamento bibliográfico sistemático até o ano de 2021 na base de dados PubMed, Cochrane e Science Direct. Cujo critério de exclusão foram publicações que não relataram associação entre plexo braquial e sequência de difusão em ressonância magnética. A revisão de literatura aponta que a técnica de difusão (DWI) por ressonância magnética (RM) demonstraram imagens satisfatoriamente seletivas para visualização anatômica do plexo braquial e alta intensidade de sinal das raízes nervosas e nervos periféricos, proporcionando detalhamento da topografia e descrevendo a longa trajetória do plexo braquial, permitindo uma avaliação mais exata e segura quando comparada com as imagens planares convencionais. Os estudos demonstraram a importância da Neurografia por difusão em RM como método promissor para visualização de nervos periféricos na região de plexo braquial.
\end{abstract}

Palavras-chave: Plexo braquial; Ferimentos e lesões; Imagem de difusão por ressonância magnética; Avaliação de danos.

\begin{abstract}
The brachial plexus is formed by the union of the ventral branches of the C5-T1 roots and, occasionally, of the C4 and T2 roots, where the most frequent causes of traumatic injuries involve traffic accidents, mainly collisions by motorcycles. The precise diagnosis of the level of the lesion and degree of impairment is of fundamental prognostic importance and determines the therapeutic approach for each clinical case. In order to evaluate the limitations and perspectives of the application of diffusion neurography in MRI for generating brachial plexus images in different pathological situations, a systematic bibliographic survey was carried out until the year 2021 in the PubMed,
\end{abstract}


Cochrane and Science Direct database. The exclusion criteria of which were publications that did not report an association between brachial plexus and diffusion sequence on magnetic resonance imaging. The literature review points out that the diffusion technique (DWI) by magnetic resonance imaging (MRI) demonstrated satisfactory selective images for anatomical visualization of the brachial plexus and high signal intensity of the nerve roots and peripheral nerves, providing details of the topography and describing the long trajectory of the brachial plexus, allowing a more accurate and safe evaluation when compared with conventional planar images. The studies demonstrated the importance of diffusion neurography in MRI as a promising method for visualizing peripheral nerves in the brachial plexus region.

Keywords: Brachial plexus; Wounds and injuries; Diffusion magnetic resonance imaging; Damage assessment.

\section{Resumen}

El plexo braquial está formado por la unión de las ramas ventrales de las raíces C5-T1 y, en ocasiones, de las raíces C4 y T2, donde las causas más frecuentes de lesiones traumáticas son los accidentes de tráfico, fundamentalmente colisiones de motocicletas. El diagnóstico preciso del nivel de la lesión y el grado de deterioro es de fundamental importancia pronóstica y determina el abordaje terapéutico de cada caso clínico. Con el fin de evaluar las limitaciones y perspectivas de la aplicación de la neurografía de difusión en resonancia magnética para la generación de imágenes del plexo braquial en diferentes situaciones patológicas, se realizó un levantamiento bibliográfico sistemático hasta el año 2021 en la base de datos PubMed, Cochrane y Science Direct. Los criterios de exclusión fueron las publicaciones que no informaron una asociación entre el plexo braquial y la secuencia de difusión en la resonancia magnética. La revisión de la literatura muestra que la técnica de difusión (DWI) por resonancia magnética (MRI) demostró imágenes selectivas satisfactorias para la visualización anatómica del plexo braquial y alta intensidad de señal de las raíces nerviosas y nervios periféricos, proporcionando detalles de la topografía y describiendo el largo trayectoria del plexo braquial, lo que permite una evaluación más precisa y segura en comparación con las imágenes planas convencionales. Los estudios han demostrado la importancia de la neurografía de difusión en la resonancia magnética como un método prometedor para visualizar los nervios periféricos en la región del plexo braquial.

Palabras clave: Plexo braquial; Heridas y traumatismos; Imagen de difusión por resonancia magnética; Evaluación de daños.

\section{Introdução}

O plexo braquial é formado pela união dos ramos ventrais das raízes a partir das vertebras C5-T1, com participação ocasional de C4 e T2 e atravessam os músculos escaleno anterior e médio. Assim, raízes nervosas C5 e C6 unem-se adjacentes à borda lateral do músculo escaleno anterior para formar o tronco superior do plexo braquial, a raiz originada a partir da $\mathrm{C} 7$ isoladamente constitui o tronco médio, enquanto as raízes C8 e T1 formam posteriormente ao músculo escaleno anterior, o tronco inferior, que se situa sobre a primeira costela (Hassan, Bassiouny \& Mohammad, 2018; Hozgrefe et al., 2019; Narahashi et al., 2005).

Dessa forma, o plexo braquial é uma rede complexa formada por raízes e nervos periféricos e, assim, uma compreensão completa das relações anatômicas normais desta estrutura é essencial para a interpretação de seus processos patológicos e lesões traumáticas. E os acidentes de trânsito, sobretudo os que envolvem colisão por motocicletas, são as causas mais frequentes de lesões por trauma em plexo braquial (Limeira, Mingueti \& Seixas, 2001; Zhang et al., 2008).

Dessa forma, um diagnóstico preciso do nível da lesão e grau de comprometimento tem fundamental importância prognóstica e determina a conduta terapêutica para cada caso clínico ${ }^{(3)}{ }^{(1)}$. Por isso, a ressonância magnética pode fornecer avaliação estrutural detalhada da região do plexo, suas raízes nervosas e ramos dos nervos periféricos (Zhang et al., 2008).

O advento da Ressonância Magnética (RM) tem proporcionado uma avaliação multiplanar e tridimensional não invasiva, permitindo a visualização de imagens de alta resolução da região do plexo braquial, sendo apontada como método de primeira escolha no diagnóstico destas plexopatias (Hozgrefe et al., 2019; Limeira, Mingueti \& Seixas, 2001; Nouguerol \& Barousse, 2020)

A indicação mais frequente de realização de RM do plexo braquial são os traumas, que podem provocar lesão de seus componentes por mecanismo de tração, por trauma direto fechado, por compressão ocasionada por hematomas ou tecidos adjacentes lesados (Narahashi et al., 2005). E com avanço na geração de imagens 3D, melhores técnicas de supressão de gordura, projetos de bobinas receptoras com sinais e, consequentemente, resoluções superiores, o aumento da disponibilidade 
da técnica somada ao uso de equipamentos com campo magnéticos superiores favorecem uma melhor visualização da complexidade do plexo braquial (Chhabra et al., 2013).

A neurografia por ressonância magnética (NRM) é uma nova técnica para a avaliação de doenças do sistema nervoso periférico. A literatura não contempla publicações em larga escala de resultados referentes ao uso de NRM para avaliação de plexopatias braquiais (Hassan, Bassiouny \& Mohammad, 2018; Hiwatashi et al., 2018; Nouguerol \& Barousse, 2020; Sarikaya et al., 2005; Zare et al., 2018).

Portanto, o objetivo desse estudo é a partir de uma revisão da literatura investigar a utilização da neurografia por difusão em RM para geração de imagens do plexo braquial. Os dados foram descritos e discutidos, a fim de avaliar a limitação e também as perspectivas da aplicação deste método em diferentes situações patológicas que podem acometer o plexo braquial.

\section{Metodologia}

A presente revisão se baseou em uma busca sistemática, baseado no estudo de Sousa, Silva e Carvalho (2010), de artigos publicados até o ano de 2021, a fim de identificar informações suficientes e claras sobre sequência de difusão em RM e plexo braquial, além da respectiva associação de ambas.

As pesquisas foram realizadas utilizando os bancos de dados da PubMed, Cochrane e Science Direct usando as palavras-chave diffusion-weighted MR neurography and brachial plexus. Dessa maneira, 58 artigos foram encontrados e após uma análise crítica dos títulos e resumos, 37 artigos foram excluídos por não estarem totalmente disponíveis e/ou aqueles que satisfaziam o objetivo do presente estudo.

\section{Resultados e Discussão}

O plexo braquial pode ser afetado por uma infinidade de patologias resultando em complicações muitas vezes graves e incapacitantes. E uma visualização radiológica mais detalhada tem grande potencial no auxílio aos médicos na garantia de prestação de serviços de qualidade para estes pacientes. A diferenciação clínica de plexopatias braquiais de outras anomalias relacionadas a coluna vertebral muitas vezes torna-se um desafio e os eletroneurodiagnósticos são de difícil execução devido a localização anatômica profunda do plexo, muitas vezes levando a resultados inconclusivos. A RM tem sido cada vez mais utilizada nos últimos anos para promover avaliação dos casos de suspeita de plexopatias braquiais com excelentes resultados (Chhabra et al., 2013).

Os nervos periféricos não podem ser visualizados seletivamente nas imagens convencionais de RM, tais como as ponderadas em T1 e T2, devido a semelhança da intensidade de sinal dos nervos periféricos e estruturas circundantes (Hozgrefe et al., 2019). A técnica de neurografia por RM é baseada em um conceito desenvolvido recentemente de imagem ponderada por difusão que demonstra tecidos com tempo de relaxamento T2 relativamente longo e difusão restrita, tais como cérebro, medula espinhal e nervos periféricos (Zare et al., 2018). Ainda, a utilização de pulso de recuperação de inversão que atua suprimindo a gordura sólida e a ponderação pesada em difusão garantem, desta forma, a supressão de sinais indesejados como os de líquidos livres, gorduras, músculos, tendões e vasos sanguíneos (Takahara et al., 2010; Yamashita et al., 2009).

As imagens ponderadas em difusão (DWI) mostram áreas com difusão restrita da água extracelular, como o tecido infartado. Nos tecidos normais a água extracelular difunde-se de forma aleatória, enquanto nos tecidos isquêmicos as células tornam-se tumefeitas e absorvem água e, assim, reduzem a difusão média. Na DWI a sequência pode ser sensibilizada para difusão por meio da aplicação de gradientes iguais de cada lado de um pulso de radiofrequência de $180^{\circ}$ (Hozgrefe et al., 2019; Nouguerol \& Barousse, 2020; Westbrook, 2010).

A neurografia por RM, técnica nervo-seletiva, visa suprimir o sinal vascular e gorduroso gerando imagens mais exclusivas para nervos periféricos, úteis na avaliação médica radiológica. A técnica deve ser incorporada ao protocolo sempre 
que a localização precisa de nervos periféricos é requerida, bem como em casos de avaliação pré-cirúrgica. Os nervos periféricos têm seus componentes estruturais organizados longitudinalmente proporcionando difusão anisotrópica. Desta forma, a adição da sequência ponderada por difusão direcionalmente codificada, gera imagens por seleção longitudinal dos nervos periféricos em decorrência da predominação e difusibilidade longitudinal de prótons (Hassan, Bassiouny \& Mohammad, 2018; Westbrook, 2010; Zare et al., 2018).

Teoricamente, o posicionamento perpendicular do gradiente aplicado na sequência de difusão (DWI) oferece maior seletividade dos nervos periféricos, uma vez que a difusão é relativamente mais restrita perpendicularmente ao eixo dos mesmos (Chhabra et al., 2013; Nouguerol \& Barousse, 2020; Takahaa et al., 2008).

A neurografia por DWI MR é capaz de descrever a longa trajetória do plexo braquial com alta conspicuidade. Quando comparada com imagens ponderadas por recuperação de inversão (STIR), a DWI é superior, pois imagens STIR valorizam estruturas circunvizinhas como líquor e vasos sanguíneos, não proporcionando clara conspicuidade do plexo braquial (Takahara et al., 2008; Zare et al., 2018). A especificação exata quanto à localização de uma lesão de raiz nervosa pode ter implicações terapêuticas importantes na conduta médica e consequentemente um melhor prognóstico clínico para estes pacientes acometidos por distúrbios do plexo braquial (Zare et al., 2018).

Os tumores secundários envolvendo o plexo braquial são mais comuns do que os tumores neurogênicos primários. A plexopatia braquial decorrente de tumores malignos mais frequentes surgem de carcinomas de mama ou pulmão. Na prática oncológica, a distinção fundamental é entre recidiva tumoral e condições benignas como fibrose e inflamação resultantes, muitas vezes, de tratamentos como quimioterapia, radioterapia e cirurgias. Os resultados obtidos em estudo com pacientes oncológicos demonstraram que a visualização anatômica da combinação da RM convencional com adição da difusão (DWMRN) melhorou significativamente a visualização anatômica do plexo braquial e esta melhoria foi observada em todos os níveis dos plexos braquiais avaliados (Andreou et al., 2015).

O Coeficiente Aparente de Difusão (ADC) é a medida da difusividade ou mobilidade microscópica dos prótons da água nos tecidos e sua unidade de medida é expressa $\mathrm{em} \mathrm{m}^{2} / \mathrm{s}$. A diferença nos valores de ADC nas lesões ocorrem provavelmente devido fatores como celularidade do tumor, integridade de membranas celulares e nucleares, proporção citoplasmática e teor de água extracelular na matriz tecidual. Estas têm sido as hipóteses postuladas para explicar as baixas taxas de difusividade observadas no interior de tumores sólidos malignos através de estudos prévios realizados (Nouguerol \& Barousse, 2020; Yuh et al., 2015).

Foi demonstrado que as imagens DWI-MRN são capazes de distinguir lesões benignas e lesões malignas através de medidas de valores de difusividade, Coeficiente Aparente de Difusão (Apparent Diffusion Coefficient - ADC), realizadas através de seleção de áreas específicas em lesões e tecidos demonstrados nestas imagens de ressonância magnética ponderadas por difusão. Existe uma diferença estatisticamente significante entre valores de ADC para as lesões encontradas em região de plexo braquial, onde lesões malignas demonstraram valores de $\mathrm{ADC} \leq 1.08 \times 10^{-3} \mathrm{~mm}^{2} / \mathrm{s}$ e lesões benignas apresentaram valores de $\mathrm{ADC} \geq 1.30 \times 10^{-3} \mathrm{~mm}^{2} / \mathrm{s}$ (Yuh et al., 2015).

A difusão é anisotrópica no sistema nervoso, incluindo nervos periféricos, e é mais restrita perpendicularmente ao eixo dos nervos, ao passo que a maior difusividade pode ser esperada paralelamente ao longo eixo destas estruturas. Assim, uma maior intensidade de sinal dos nervos periféricos pode ser obtida através da aplicação de apenas um par de movimento de sondagem gradiente (MPGs), perpendicular ao curso dos nervos, ao passo que a intensidade do sinal dos nervos periféricos é mais baixa quando se aplica o gradiente paralelamente ao curso nervoso. Por outro lado, muitas estruturas adjacentes (gânglios linfáticos, medula óssea, vasos sanguíneos de fluxo lento) sempre exibem alta intensidade de sinal, independentemente da direção de gradiente aplicada (Takahara et al., 2011). 
A visualização seletiva de nervos periféricos pode ser alcançada fazendo-se uso de técnica de subtração DW-MRN, onde trabalha-se, a princípio, com um conjunto de dados obtidos mediante aplicação paralela de apenas um par de MPGs ao longo dos nervos e posteriormente adquire-se um outro conjunto de dados DW-MRN obtido através de aplicação de um par de MPGs perpendicular ao curso de os nervos. Este é o princípio da técnica proposta pela modalidade de difusão SUSHI (Takahara et al., 2011).

Há também um novo método proposto de aquisição rápida de imagens neurográficas de alta resolução utilizando sequência tridimensional turbo spin eco combinada com pré-pulso de difusão denominada Improved motion-sensitized driven equilibrium (iMSDE), denominada Diffusion-prepared MR Neurography (D-prep MRN), na qual fazendo-se uso de valor de $b$ de $10 \mathrm{~s} / \mathrm{mm}^{2}$ houve melhor supressão de vasos sanguíneos, enquanto que um iMSDE prep-time intermediário de $50 \mathrm{~ms}$ proporcionou melhores resultados entre supressão de sinal dos músculos e minimização do baixo sinal dos nervos. Com estes parâmetros, as estruturas nervosas apresentaram elevada intensidade de sinal, enquanto que o músculo e vasos sanguíneos foram efetivamente suprimidos. Os resultados demonstram, consistentemente, imagens de alta resolução com excelente relação sinal-ruído (SNR) e contraste entre estruturas nervosas e tecidos adjacentes. Consequentemente, a sequência D-prep MRN otimizada mostrou claramente os detalhes anatômicos e a trajetória tridimensional do plexo braquial (Yoneyama et al., 2013).

Estudos que comparam imagens por difusão do plexo braquial adquiridas em equipamentos com intensidade de campos magnéticos diferentes ressaltam que as imagens adquiridas nas máquinas de $1.5 \mathrm{~T}$ são mais satisfatórias quando comparadas com as imagens produzidas pelos equipamentos de 3.0 T. Parte disto está relacionado a sobreposição de estruturas ao plexo nervoso, como linfonodos, líquor e estruturas vasculares, fenômeno este que é maior especialmente na região do pescoço avaliada por equipamentos de 3.0 T. O contraste entre nervos e estruturas adjacentes indesejadas varia de acordo com a força do campo magnético. Sabemos ainda que máquinas de maior amplitude de campo magnético (3.0 T) são mais suscetíveis a artefatos de susceptibilidade magnética, bem como artefatos de fluxo e movimentos. Como exemplos podemos citar a presença de ar em cavidades e órgãos, batimentos cardíacos, respiração, fluxo sanguíneo e liquórico (Mutz et al., 2015; Nouguerol \& Barousse, 2020).

O uso de um amplo campo de visão (FOV) durante a aquisição das imagens de plexo braquial tem grande importância clínica para deteç̧ão de anormalidades na região dos ombros e realização de comparação anatomomorfológica contralateral. O uso de estruturas como travesseiros que acomodem o paciente e minimizem diferenças de susceptibilidade entre o ar e o tecido ao redor do pescoço, diminuindo consideravelmente a presença de artefatos e melhorando a homogeneidade do campo magnético na região do plexo braquial, contribuem para melhor qualidade e eficiência das imagens por difusão, principalmente em equipamentos de elevado campo magnético, como os aparelhos de 3.0 T em uso clínico atualmente (Oudeman et al., 2016).

Todos os estudos analisados demonstram que a técnica de difusão (DWI) por ressonância magnética (RM) oferece imagens satisfatoriamente seletivas para visualização anatômica do plexo braquial, proporcionando a supressão de tecidos e estruturas adjacentes. Ainda, apresenta alta intensidade de sinal das raízes nervosas e nervos periféricos, além de proporcionar, através de reconstruções tridimensionais MIP (Maximum Intensity Projection) em plano coronal, detalhamento da topografia, descrevendo a longa trajetória do plexo braquial, retratando todo o comprimento das bainhas nervosas, permitindo uma avaliação mais exata e segura quando comparada com as imagens planares convencionais, tornando-se indispensável para localização e avaliação de lesões traumáticas, inflamatórias, infiltrativas, tumorais ou compressivas provenientes dos tecidos circundantes.

A Tabela 1 resumiu os artigos selecionados a marca do equipamento de ressonância magnética, potência do campo magnético, bobina receptora utilizada, canais, fator b de difusão, tipo de lesão avaliada em plexo braquial, intensidade de sinal, qualidade global da imagem, visibilidade da trajetória dos nervos do plexo braquial e visualização de lesões. Imagens ponderadas em difusão são imagens funcionais que oferecem melhor contraste de imagem entre os nervos periféricos e tecidos 
adjacentes, tais como estruturas vasculares, líquor e feixes musculares devido à restrição de difusão relativamente forte perpendicular ao longo eixo das fibras nervosas combinadas a um longo tempo de relaxamento T2 (Mutz et al., 2015).

Tabela 1 - Resumo dos artigos a respeito do uso da neurografia por difusão em RM para geração de imagens do plexo braquial.

\begin{tabular}{|c|c|c|c|c|c|c|}
\hline Referências & $\begin{array}{c}\text { Marca do } \\
\text { Fabricante do } \\
\text { Equipamento de } \\
\text { RM }\end{array}$ & $\begin{array}{l}\text { Potência do } \\
\text { Campo } \\
\text { Magnético } \\
\text { (T) }\end{array}$ & $\begin{array}{l}\text { Bobina } \\
\text { Receptora } \\
\text { Utilizada / } \\
\text { Canais }\end{array}$ & $\begin{array}{l}\text { Fator b de } \\
\text { Difusão } \\
\left(\mathbf{s} / \mathbf{m m}^{2}\right)\end{array}$ & $\begin{array}{l}\text { Tipo de lesão (quando } \\
\text { existir) avaliada em } \\
\text { plexo braquial }\end{array}$ & $\begin{array}{c}\text { Intensidade de } \\
\text { sinal/ Qualidade } \\
\text { global da imagem/ } \\
\text { Visibilidade da } \\
\text { trajetória dos } \\
\text { nervos do plexo } \\
\text { braquial/ } \\
\text { Visualização de } \\
\text { lesões (caso existam) }\end{array}$ \\
\hline $\begin{array}{l}\text { Takahara et al. } \\
\qquad(2008)\end{array}$ & Philips Gyroscan & $1.5 \mathrm{~T}$ & $\begin{array}{c}\text { Surface Coil } \\
-4\end{array}$ & 1000 & $\begin{array}{l}\text { Voluntários Saudáveis / } \\
\text { Patologias não } \\
\text { informadas }\end{array}$ & Satisfatória \\
\hline $\begin{array}{c}\text { Takahara et al. } \\
\qquad(2011)\end{array}$ & Philips Achieva & $1.5 \mathrm{~T}$ & $\begin{array}{c}\text { Surface Coil } \\
-4,8,18\end{array}$ & 900 & Não informado & Satisfatória \\
\hline $\begin{array}{l}\text { Yoneyama et } \\
\text { al. (2013) }\end{array}$ & Philips Achieva & $3.0 \mathrm{~T}$ & $\begin{array}{c}\text { Surface Coil } \\
-16\end{array}$ & 0,2 e 10 & Voluntários Saudáveis & Satisfatória \\
\hline $\begin{array}{l}\text { Andreou et al. } \\
\qquad(2015)\end{array}$ & Siemens Avanto & $1.5 \mathrm{~T}$ & $\begin{array}{c}\text { Cervical } \\
\text { Spine - } 2 \\
\text { Torso Coil - } \\
16\end{array}$ & 700 & Oncológicas & Satisfatória \\
\hline $\begin{array}{l}\text { Murtz et al. } \\
\text { (2015) }\end{array}$ & $\begin{array}{l}\text { Philips Intera } \\
\text { Philips Achieva }\end{array}$ & $\begin{array}{l}1.5 \mathrm{~T} \\
3.0 \mathrm{~T}\end{array}$ & $\begin{array}{c}\text { Sense Body - } \\
4 \text { Sense NV - } \\
16 \\
\text { Sense Torso - } \\
16 \\
\text { Sense NV - } \\
16\end{array}$ & $800-1000$ & Anormalidades nervosas & Satisfatória \\
\hline $\begin{array}{l}\text { Yuh et al. } \\
\text { (2015) }\end{array}$ & Philips Gyroscan & $1.5 \mathrm{~T}$ & $\begin{array}{c}\text { Não } \\
\text { informado }\end{array}$ & 400 & $\begin{array}{c}\text { Neuropatias } \\
\text { pseudotumorais ou } \\
\text { infiltrativas }\end{array}$ & Satisfatória \\
\hline $\begin{array}{l}\text { Oudeman et al. } \\
\qquad(2016)\end{array}$ & Philips Ingenia & $3.0 \mathrm{~T}$ & $\begin{array}{l}\text { Torso Coil + } \\
\text { Head Coil - } \\
16\end{array}$ & $\begin{array}{c}\text { Não } \\
\text { especificado }\end{array}$ & Voluntários Saudáveis & Satisfatória \\
\hline $\begin{array}{l}\text { Hassan, } \\
\text { Bassiouny, } \\
\text { Mohammad } \\
(2018)\end{array}$ & $\begin{array}{c}\text { MR System } \\
\text { Achiva-Philips }\end{array}$ & $1.5 \mathrm{~T}$ & $\begin{array}{l}\text { cervical spine } \\
\text { array coil }\end{array}$ & 0 a 800 & $\begin{array}{l}\text { Pacientes com suspeita de } \\
\text { lesões }\end{array}$ & Satisfatória \\
\hline $\begin{array}{l}\text { Hiwatashi et } \\
\text { al. (2018) }\end{array}$ & $\begin{array}{l}\text { MR Ingenia, } \\
\text { Philips }\end{array}$ & $3.0 \mathrm{~T}$ & $\begin{array}{l}\text { dStream } \\
\text { TotalSpine } \\
\text { coil. }\end{array}$ & 10 & $\begin{array}{l}\text { Pacientes com } \\
\text { polirradiculoneuropatia } \\
\text { desmielinizante } \\
\text { inflamatória crônica }\end{array}$ & Satisfatória \\
\hline $\begin{array}{l}\text { Zare et al. } \\
\quad(2018)\end{array}$ & MR Avanto & $1,5 \mathrm{~T}$ & $\begin{array}{l}\text { body matrix } \\
\text { coil, neck } \\
\text { matrix coil, } \\
\text { head matrix } \\
\text { coil, and CP } \\
\text { flexible coil }\end{array}$ & 90 a 100 & Voluntários saudáveis & Satisfatória \\
\hline
\end{tabular}


Fonte: Autores.

\section{Conclusão}

Em conclusão, os estudos selecionados demonstraram que a Neurografia por difusão em RM tem emergido como método promissor para visualização de nervos periféricos como na região de plexo braquial. A adição de uma sequência ponderada em difusão no protocolo do exame de RM convencional permite superar as desvantagens inerentes a RM convencional no que tange a avaliação de lesões traumáticas, inflamatórias, infiltrativas, tumorais ou compressivas provenientes dos tecidos circundantes.

Contudo, novos estudos deverão ser realizados para melhorar ainda mais a compreensão sobre essa ferramenta e suas vantagens para, assim, haver um melhor auxilio no diagnóstico de alterações no plexo braquial. Além de novos métodos que podem ser descobertos a partir da ressonância magnética.

\section{Referências}

Andreou, A., Sohaib, A., Collins, D. J., Takahara, T., Kwee, T. C., Leach, M. O., MacVicar, D. A., \& Koh, D. M. (2015). Diffusion-weighted MR neurography for the assessment of brachial plexopathy in oncological practice. Cancer imaging: the official publication of the International Cancer Imaging Society, 15(1), 6. https://doi.org/10.1186/s40644-015-0041-5

Chhabra, A., Thawait, G. K., Soldatos, T., Thakkar, R. S., Del Grande, F., Chalian, M., \& Carrino, J. A. (2013). High-resolution 3T MR neurography of the brachial plexus and its branches, with emphasis on 3D imaging. AJNR. American journal of neuroradiology, 34(3), 486-497. https://doi.org/10.3174/ajnr.A3287

Chhabra, A., Zhao, L., Carrino, J. A., Trueblood, E., Koceski, S., Shteriev, F., Lenkinski, L., Sinclair, C. D., \& Andreisek, G. (2013). MR Neurography: Advances. Radiology research and practice, 2013, 809568. https://doi.org/10.1155/2013/809568

Hassan, H. G. E. M. A., Bassiouny, R. H., \& Mohammad, S. A. (2018). Quantitative MR neurography of brachial plexus lesions based on diffusivity measurements. The Egyptian Journal of Radiology and Nuclear Medicine, 49(4), 1093-1102. https://doi.org/10.1016/j.ejrnm.2018.05.005

Hiwatashi, A., Togao, O., Yamashita, K., Kikuchi, K., Momosaka, D., Nakatake, H., Yamasaki, R., Ogata, H., Yoneyama, M., Kira, J. I., \& Honda, H. (2019). Simultaneous MR neurography and apparent T2 mapping in brachial plexus: Evaluation of patients with chronic inflammatory demyelinating polyradiculoneuropathy. Magnetic resonance imaging, 55, 112-117. https://doi.org/10.1016/j.mri.2018.09.025

Holzgrefe, R. E., Wagner, E. R., Singer, A. D., \& Daly, C. A. (2019). Imaging of the Peripheral Nerve: Concepts and Future Direction of Magnetic Resonance Neurography and Ultrasound. The Journal of hand surgery, 44(12), 1066-1079. https://doi.org/10.1016/j.jhsa.2019.06.021

Limeira, A. C. B., Minguetti, G., \& Seixas, R. (2001). Ressonância magnética na avaliação da plexopatia braquial pós-traumática. Rev. bras. ortop, 36(3), 7178. https://pesquisa.bvsalud.org/portal/resource/pt/lil-334902

Martín Noguerol, T., \& Barousse, R. (2020). Update in the evaluation of peripheral nerves by MRI, from morphological to functional neurography. Actualización en la valoración de los nervios periféricos mediante resonancia magnética: de la neurografía morfológica a la funcional. Radiologia, 62(2), 90101. https://doi.org/10.1016/j.rx.2019.06.005

Mürtz, P., Kaschner, M., Lakghomi, A., Gieseke, J., Willinek, W. A., Schild, H. H., \& Thomas, D. (2015). Diffusion-weighted MR neurography of the brachial and lumbosacral plexus: 3.0 T versus 1.5 T imaging. European journal of radiology, 84(4), 696-702. https://doi.org/10.1016/j.ejrad.2015.01.008

Narahashi, E., Caldana, W. C. I., Zoner, C., Honda, E., Caporrino, F. A., Mine, F., Yamada, D. T., Natour, J. \& Fernandes, A. D. R. C. (2005). Image diagnosis of brachial plexus. Revista Brasileira de Reumatologia, 45(4), 245-249. https://www.scielo.br/pdf/rbr/v45n4/v45n4a09.pdf

Oudeman, J., Coolen, B. F., Mazzoli, V., Maas, M., Verhamme, C., Brink, W. M., Webb, A. G., Strijkers, G. J., \& Nederveen, A. J. (2016). Diffusionprepared neurography of the brachial plexus with a large field-of-view at 3T. Journal of magnetic resonance imaging: JMRI, 43(3), 644-654. https://doi.org/10.1002/jmri.25025

Sarikaya, S., Sumer, M., Ozdolap, S., \& Erdem, C. Z. (2005). Magnetic resonance neurography diagnosed brachial plexitis: a case report. Archives of physical medicine and rehabilitation, 86(5), 1058-1059. https://doi.org/10.1016/j.apmr.2004.08.003

Souza, M. T. D., Silva, M. D. D., \& Carvalho, R. D. (2010). Revisão integrativa: o que é e como fazer. Einstein (São Paulo), 8(1), 102-106. http://dx.doi.org/10.1590/s1679-45082010rw1134

Takahara, T., Hendrikse, J., Yamashita, T., Mali, W. P., Kwee, T. C., Imai, Y., \& Luijten, P. R. (2008). Diffusion-weighted MR neurography of the brachial plexus: feasibility study. Radiology, 249(2), 653-660. https://doi.org/10.1148/radiol.2492071826

Takahara, T., Kwee, T. C., Hendrikse, J., Van Cauteren, M., Koh, D. M., Niwa, T., Mali, W. P., \& Luijten, P. R. (2011). Subtraction of unidirectionally encoded images for suppression of heavily isotropic objects (SUSHI) for selective visualization of peripheral nerves. Neuroradiology, 53(2), 109-116. https://doi.org/10.1007/s00234-010-0713-6 
Research, Society and Development, v. 10, n. 5, e36810515149, 2021

(CC BY 4.0) | ISSN 2525-3409 | DOI: http://dx.doi.org/10.33448/rsd-v10i5.15149

Takahara, T., Kwee, T. C., Van Leeuwen, M. S., Ogino, T., Horie, T., Van Cauteren, M., Herigault, G., Imai, Y., Mali, W. P., \& Luijten, P. R. (2010). Diffusion-weighted magnetic resonance imaging of the liver using tracking only navigator echo: feasibility study. Investigative radiology, 45(2), 57-63. https://doi.org/10.1097/RLI.0b013e3181cc25ed

Westbrook, C. (2010). Manual de técnicas de Ressonância Magnética (3nd ed.). Guanabara Koogan.

Yamashita, T., Kwee, T. C., \& Takahara, T. (2009). Whole-body magnetic resonance neurography. The New England journal of medicine, 361(5), 538-539. https://doi.org/10.1056/NEJMc0902318

Yoneyama, M., Takahara, T., Kwee, T. C., Nakamura, M., \& Tabuchi, T. (2013). Rapid high resolution MR neurography with a diffusion-weighted prepulse. Magnetic resonance in medical science.s MRMS: an official journal of Japan Society of Magnetic Resonance in Medicine, 12(2), 111-119. https://doi.org/10.2463/mrms.2012-0063

Yuh, E. L., Jain Palrecha, S., Lagemann, G. M., Kliot, M., Weinstein, P. R., Barbaro, N. M., \& Chin, C. T. (2015). Diffusivity measurements differentiate benign from malignant lesions in patients with peripheral neuropathy or plexopathy. AJNR. American journal of neuroradiology, 36(1), 202-209. https://doi.org/10.3174/ajnr.A4080

Zare, M., Faeghi, F., Hosseini, A., Ardekani, M. S., Heidari, M. H., \& Zarei, E. (2018). Comparison Between Three-Dimensional Diffusion-Weighted PSIF Technique and Routine Imaging Sequences in Evaluation of Peripheral Nerves in Healthy People. Basic and Clinical Neuroscience, 9(1), 65-71. https://doi.org/10.29252/NIRP.BCN.9.1.65

Zhang, Z., Song, L., Meng, Q., Li, Z., Luo, B., Pei, Z., \& Zeng, J. (2008). Segmented echo planar MR imaging of the brachial plexus with inversion recovery magnetization preparation at 3.0T. Journal of magnetic resonance imaging: JMRI, 28(2), 440-444. https://doi.org/10.1002/jmri.21304 